Canadian

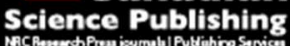

Applied Physiology, Nutrition, and Metabolism Physiologie appliquée, nutrition et métabolisme

\title{
Maximal power output during incremental cycling test is dependent on the curvature constant of the power-time relationship
}

\begin{tabular}{|r|l|}
\hline Journal: & Applied Physiology, Nutrition, and Metabolism \\
\hline Manuscript ID: & apnm-2015-0090.R2 \\
\hline Manuscript Type: & Article \\
\hline Date Submitted by the Author: & 22-Apr-2015 \\
\hline Complete List of Authors: & $\begin{array}{l}\text { Souza, Kristopher; Federal University of Santa Catarina, Sports Center } \\
\text { de Lucas, Ricardo; Federal University of Santa Catarina, Sports Center } \\
\text { do Nascimento Salvador, Paulo; Federal University of Santa Catarina, } \\
\text { Sports Center } \\
\text { Guglielmo, Luiz; Federal University of Santa Catarina, Sports Center } \\
\text { Caritá, Renato; Human Performance Laboratory, } \\
\text { Greco, Camila; Human Performance Laboratory, } \\
\text { Denadai, Benedito; Human Performance Laboratory, }\end{array}$ \\
\hline Keyword: & $\begin{array}{l}\text { exercise performance < exercise, cycling < sports, endurance, fatigue < } \\
\text { exercise, critical power }\end{array}$ \\
\hline
\end{tabular}


1 Maximal power output during incremental cycling test is dependent on the

2 curvature constant of the power-time relationship

3

4 Kristopher Mendes de Souza², Ricardo Dantas de Lucas², Paulo Cesar do

5 Nascimento Salvador ${ }^{2}$, Luiz Guilherme Antonacci Guglielmo², Renato Aparecido

6 Corrêa Caritá ${ }^{1}$, Camila Coelho Greco ${ }^{1}$, Benedito Sérgio Denadai ${ }^{1}$

7

81 - Human Performance Laboratory, UNESP, Rio Claro, Brazil

92 - Physical Effort Laboratory, Sports Center, Federal University of Santa Catarina,

10 Florianópolis, Brazil

11

12 Benedito Sérgio Denadai $(\square)$

13 Human Performance Laboratory, IB - UNESP, Rio Claro, São Paulo, Brasil.

14 Avenida 24 A, 1515, Bela Vista - CEP 13506-900. e-mail - bdenadai@rc.unesp.br 15 


\section{Abstract}

2 The aim of this study was to investigate whether the maximal power output

$3\left(P_{\max }\right)$ during an incremental test (INC) was dependent on the curvature constant

4 (W') of the power-time relationship. Thirty healthy male subjects $\left(\mathrm{VO}_{2 \max }=3.58 \pm\right.$

$5 \quad 0.40$ L. $\min ^{-1}$ ) performed a ramp incremental cycling test to determine the $\mathrm{VO}_{2 \max }$

6 and $P_{\max }$, and four constant work rate tests to exhaustion in order to estimate two

7 parameters from the modeling of the power-time relationship (i.e., critical power -

8 CP and W'). Afterwards, the participants were ranked according to their magnitude

9 of W'. The median third was excluded to form a high W' group ( $\mathrm{HIGH}, \mathrm{n}=10)$, and

10 a low W' group (LOW, $n=10)$. $\mathrm{VO}_{2 \max }\left(3.84 \pm 0.50\right.$ vs. $\left.3.49 \pm 0.37{\mathrm{~L} . \mathrm{min}^{-1}}^{-1}\right)$ and CP

$11(213 \pm 22$ vs. $200 \pm 29$ W) were not significantly different between HIGH and LOW

12 respectively. However, $\mathrm{P}_{\max }$ was significantly greater for the $\mathrm{HIGH}(337 \pm 23 \mathrm{~W})$

13 than for the LOW $(299 \pm 40 \mathrm{~W})$. Thus, in physically active individuals with similar

14 aerobic parameters, $W^{\prime}$ influences the $P_{\max }$ during INC.

15 Key words: exercise performance, cycling, endurance, fatigue, critical power, 16 ramp incremental test. 


\section{Introduction}

$2 \quad$ Maximal oxygen uptake $\left(\mathrm{VO}_{2 \max }\right)$ and maximal power output $\left(\mathrm{P}_{\max }\right)$, both 3 determined during ramp or step incremental tests (INC), have been used 4 extensively for training prescription (Green et al. 2013) and endurance 5 performance prediction (McNaughton et al. 2006). $P_{\max }$ is influenced by both the 6 physiological parameters (e.g., exercise economy, anaerobic capacity and muscle 7 power) (Jones and Carter 2000), and exercise protocols used for its determination 8 (Bentley and McNaughton 2003). There is a consensus that while $\mathrm{VO}_{2 m a x}$ remains 9 unchanged, the $\mathrm{P}_{\max }$ is protocol-dependent (i.e., ramp slopes or step increments and durations) (Bentley and McNaughton 2003). Overall, it has been shown that

11 the ramp incremental tests result in greater $P_{\max }$ than that attained during the step 12 incremental tests (Zuniga et al. 2012), and that steeper ramps elicit higher $P_{\max }$ 13 (Morton 2011).

14 On the other hand, the identification of the factors influencing the inter15 individual variability of $P_{\max }$ remains a topic of intense debate. It has been 16 proposed that $\mathrm{P}_{\max }$ reflects the association between $\mathrm{VO}_{2 \max }$ and exercise economy 17 (Billat et al. 2003). However, Rønnestad et al. (2014) found in a group of elite 18 cyclists that $P_{\max }$ was increased after heavy strength training, while no significant 19 change was observed in $\mathrm{VO}_{2 \max }$ and gross efficiency. Thus, other metabolic (e.g., 20 anaerobic capacity) and neuromuscular (e.g., muscle power) variables have been 21 also associated with $P_{\max }$ (Jones and Carter 2000), although direct evidence for 22 this relationship is limited or equivocal.

23 Regarding these likely associations, some insights can be obtained from 24 the critical power concept. Exercise tolerance (Tlim) during high-intensity exercise 25 can be predicted by the curvature constant $\left(\mathrm{W}^{\prime}\right)$ of the power-time relationship 
1 (Jones et al. 2010). The asymptote of this relationship, termed critical power (CP),

2 is considered to be the lower boundary of the severe-intensity domain (equation $31)$.

$$
T \lim =W^{\prime} /(P-C P)
$$

5 where $\mathrm{P}$ represents the power output above CP. Evidence shows that W' dictates

6 the Tlim during severe-intensity exercise, with exercise intolerance coinciding with

7 the accumulation of metabolites that are linked to the process of muscle fatigue

8 until some critical concentration is attained (i.e., $[\mathrm{PCr}],\left[\mathrm{P}_{\mathrm{i}}\right]$, and $\left.\left[\mathrm{H}^{+}\right]\right)($Vanhatalo et

9 al. 2010). Appling the CP model (CP and W' as constants and the ramp slope as

10 variable) to INC with different ramp slopes, Morton (2011) has demonstrated why

11 steeper ramps determining higher $\mathrm{P}_{\max }$ according to equation 2 .

$$
T \lim =C P / S+\operatorname{sqrt}\left(2 W^{\prime} / S\right)
$$

13 where S represents the ramp slope. Indeed, considering that W' represents a fixed

14 amount of work that can be performed above $\mathrm{CP}$, irrespective of the rate of its 15 expenditure (Jones et al. 2010), steeper ramps, and consequently, lower time 16 above $\mathrm{CP}$, can determine higher $\mathrm{P}_{\max }$ (Morton 2011). According to this data, the 17 magnitude of $W^{\prime}$ should be able to discriminate the $\mathrm{P}_{\max }$ of individuals with similar 18 CP values. Thus, a likely factor that determines $P_{\max }$ during INC is therefore W', 19 although this has never been directly verified.

20 Therefore, our main objective was to compare the $\mathrm{P}_{\max }$ between two groups 21 having similar CP but different W'. For this purpose, participants were ranked 22 according to their W' and the median third was excluded to form a low W' group 23 (LOW) and a high W' group (HIGH). It was hypothesized that the higher the W', 24 the higher the $\mathrm{P}_{\max }$ obtained during INC. In addition, the correlations between $\mathrm{P}_{\max }$ 25 and variables derived from INC and CP model were analyzed. 


\section{Material and methods}

\section{Subjects}

Thirty healthy male subjects (mean \pm SD; age, $25.9 \pm 3.7$ years; weight,

$5 \quad 77.5 \pm 8.8 \mathrm{~kg}$; height, $177.4 \pm 6.6 \mathrm{~cm}$ ) volunteered to participate in this study. The

6 subjects participated in exercise at a recreational level and were familiar with cycle

7 ergometry and exercise testing procedures used in our laboratory. After being fully

8 informed of the risks and stresses associated with the study, the subjects gave

9 their written informed consent to participate. The experimental protocol was approved by the local Ethics Committee of the University and was conducted in

11 accordance with the Declaration of Helsinki.

\section{Study design}

14 The subjects were required to visit the laboratory on five different 15 occasions. Each subject performed the following testing stages: 1) a submaximal 16 step incremental test (four to five work rates) to determine the lactate threshold

17 (LT), followed by a maximal ramp incremental test for the measurement of $\mathrm{VO}_{2 \max }$ 18 and $\mathrm{P}_{\max }$; and; 2) four maximal constant work rate tests performed to exhaustion at $1975 \%, 85 \%, 95 \%$ and $105 \% \mathrm{P}_{\max }$ for $\mathrm{CP}$ and $\mathrm{W}^{\prime}$ determination. The subjects were 20 instructed to avoid any intake of caffeine or alcohol and strenuous exercise in the $2124 \mathrm{~h}$ preceding a test session and to arrive at the laboratory in a rested and fully 22 hydrated state, at least $3 \mathrm{~h}$ postprandial. All tests were performed at the same time 23 of day in a controlled environmental laboratory condition $\left(19-22^{\circ} \mathrm{C} ; 50-60 \% \mathrm{RH}\right)$ to 24 minimize the effects of diurnal biological variation on the results. With exception of 25 the submaximal and maximal incremental exercise tests, which were performed on 
1 the same day, the subjects performed only one test on any given day, and the

2 tests were each separated by $24-48 \mathrm{~h}$ but completed within a period of two weeks.

3 Upon study completion, the participants were ranked according to their magnitude

4 of W'. The median third was excluded to form a high W' group ( $\mathrm{HIGH}, \mathrm{n}=10)$, and

5 a low W' group (LOW, $n=10)$. There was no overlap in the W' values between the 6 groups.

\section{Equipment}

10 (Excalibur Sport, Lode BV, Groningen, Netherlands). For all stages, pedal

11 cadence was selected at $70 \pm 1 \mathrm{rpm}$. Marsh and Martin (1997) reported that

12 individuals without cycling training maintained a preferred pedal cadence between

1365 and $80 \mathrm{rpm}$. Respiratory and pulmonary gas exchange variables were

14 measured continuously using a breath-by-breath analyzer (Quark PFTergo,

15 Cosmed, Rome, Italy). Before each test, the $\mathrm{O}_{2}$ and $\mathrm{CO}_{2}$ analysis systems were

16 calibrated using ambient air and a gas of known $\mathrm{O}_{2}$ and $\mathrm{CO}_{2}$ concentration

17 according to the manufacturer's instructions, while the Quark PFTergo turbine flow

18 meter was calibrated using a $3 \mathrm{~L}$ syringe (Calibration Syringe $3 \mathrm{~L}$, Cosmed, Rome,

19 Italy). Breath-by-breath $\mathrm{VO}_{2}$ data were analyzed throughout the tests (Data

20 Management Software, Cosmed, Rome, Italy). Capillary blood samples (25 $\mu$ l)

21 were obtained from the earlobe of each subject and the blood lactate

22 concentration ([La]) was measured using an electrochemical analyzer (YSL 2700

23 STAT, Yellow Springs, Ohio, USA). The analyzer was calibrated in accordance

24 with the manufacturer's recommended procedures. 


\section{Submaximal and maximal incremental tests}

2 Initially, each subject performed a submaximal step incremental test to 3 determine LT. The test started at $60 \mathrm{~W}$ and was increased by $20 \mathrm{~W}$ every $3 \mathrm{~min}$ 4 during four to five stages. Capillary blood samples were collected within the final $520 \mathrm{~s}$ of each stage for an immediate [La] determination. The LT was determined 6 from the relationship between [La] and work rate and was considered as the first 7 sudden and sustained increase in [La] above resting concentrations (Carter et al. 8 2000). After 30 min of resting, the subjects performed a maximal ramp incremental 9 test for the measurement of $\mathrm{VO}_{2 \max }$ and $\mathrm{P}_{\max }$. This test started at $90 \%$ of $\mathrm{LT}$ during 10 the first $4 \mathrm{~min}$ and was thereafter continuously increased by a rate of $25 \mathrm{~W} \cdot \mathrm{min}^{-1}$ 11 until the volitional exhaustion. Each subject was verbally encouraged to undertake 12 maximal effort. Breath-by-breath oxygen uptake $\left(\mathrm{VO}_{2}\right)$ data was reduced to $15 \mathrm{~s}$ 13 stationary averages and the $\mathrm{VO}_{2 \max }$ was considered as the highest average $15 \mathrm{~s}$ $14 \mathrm{VO}_{2}$ value recorded during the ramp incremental test. The $\mathrm{P}_{\max }$ was considered as 15 the highest power output attained in the ramp incremental test.

\section{Determination of CP and W'}

18 The subjects performed four maximal constant work rate tests until 19 exhaustion at $75 \%, 85 \%, 95 \%$ and $105 \% \mathrm{P}_{\max }$. These work rates were chosen to 20 induce a Tlim over a range of times between 3 and $15 \mathrm{~min}$ (Vanhatalo et al. 2010).

21 Each test started with a 5 min warm-up at LT intensity followed by a 5 min of rest.

22 Previous exercise performed at moderate-intensity domain (i.e., $\leq$ LT) does not 23 modify both the magnitude of $W^{\prime}$ and Tlim within severe-intensity domain (i.e., > 24 CP) (Wilkerson et al. 2003). Further, after $3 \mathrm{~min}$ at $20 \mathrm{~W}$ the power output was 25 adjusted to one of the previously established work rates and the subjects were 
1 instructed to perform until they were unable to maintain the required work rate.

2 Timing began when the pedal cadence reached $70 \mathrm{rpm}$ and stopped when the

3 subject could not maintain a pedal cadence of higher than $67 \mathrm{rpm}$ despite verbal

4 encouragement (Caputo and Denadai 2008). The Tlim was measured to the

5 nearest second.

\section{Data analysis}

8 Individual CP and W' estimates were derived from the four prediction trials

9 by least-squares fitting of the following regression models:

10 1) Nonlinear power output (P) vs. time to exhaustion (Tlim):

$$
T \lim =W^{\prime} /(P-C P)
$$

12 2) Linear work (W) vs. time to exhaustion (Tlim):

$$
W=(C P \times T \lim )+W^{\prime}
$$

14 3) Linear power output (P) vs. 1 / time to exhaustion (Tlim):

$$
P=\left(W^{\prime} / T \lim \right)+C P
$$

16 The CP and W' estimates from the three equations were compared in order

17 to select the best fit using the model associated with the lowest standard error for 18 CP (SEE) (Vanhatalo et al. 2010). The CP and W' were applied in equation 2 in 19 order to predict Tlim for the ramp incremental test $\left(S=0.41 \mathrm{~W} . \mathrm{S}^{-1}\right)$ (Morton 2011).

20 The $P_{\max }$ was estimated from equation 5:

$$
P_{\max }=S \times T \lim
$$

\section{Statistical analysis}

All data throughout are expressed as mean \pm SD. The Shapiro-Wilk test

25 was applied to ensure a Gaussian distribution of the data. Student's unpaired t-test 
1 was used in unpaired comparisons. Pearson's product-moment correlation

2 coefficient and stepwise multiple regression was used to determine the best

3 independent variables to predict $P_{\max }$ for the overall sample only. Paired $t$-tests

4 and Pearson's product-moment correlation coefficient were used to examine the

5 relationship between actual and predicted $P_{\max }$ for INC. Analyses were carried out

6 using SPSS (v. 20.0, Chicago, Illinois, USA). The level of significance was set at $p$ $7 \leq 0.05$

\section{Results}

\section{Submaximal and maximal incremental tests}

Maximal and submaximal variables obtained during INC are presented in

13 Table 1. The $\mathrm{VO}_{2 \max }$ and LT were not significant different between HIGH and LOW

14 groups. The $\mathrm{P}_{\max }$ was significantly greater in the HIGH than in the LOW group ( $p<$ 15 0.05).

\section{Determination of the power-time relationship}

18 There were no differences in parameter estimates derived from the three

19 fitting models. This goodness-of-fit for both nonlinear and linear regressions was 20 confirmed by $\mathrm{R}^{2}$-values $>0.98$. The $95 \% \mathrm{Cls}$ associated with the estimated 21 parameters of the power-time relationship were 2.7 to $5.1 \mathrm{~W}$ and 0.9 to $1.4 \mathrm{~kJ}$ for $22 \mathrm{CP}$ and W', respectively. The CP was not significantly different between the HIGH 23 and LOW groups. The CP relative to $\mathrm{P}_{\max }$ and $\mathrm{W}$ ' were significantly different 24 between the HIGH and LOW groups $(p<0.05)$. 
1 The actual $P_{\max }$ for INC $(317 \pm 33 \mathrm{~W}$; range $=217-383 \mathrm{~W})$ was not

2 significantly different from the predicted $P_{\max }(320 \pm 28 \mathrm{~W}$; range $=240-371 \mathrm{~W})$,

3 and these values were highly correlated $(r=0.94, p<0.001$; see Fig. 1). The

4 correlations between $\mathrm{P}_{\max }$ and $\mathrm{VO}_{2 \max }(r=0.61), \mathrm{P}_{\max }$ and $\mathrm{CP}(r=0.89)$ and

5 between $P_{\max }$ and $W^{\prime}(r=0.55)$ in the total sample were statistically significant $(p<$

6 0.05). A stepwise multiple regression analysis from the overall sample $(n=30)$

7 revealed that $80.7 \%$ of the variance for $P_{\max }$ could be explained by $C P$ alone $(F=$

8 113.0, $p<0.001$ ), and the addition of $W^{\prime}$ to the prediction equation increased it

9 significantly (partial $F=6.8, p<0.01$ ) to $89.5 \%$ of the variance. Moreover, the

10 difference between $P_{\max }$ and CP was significantly correlated with $W^{\prime}(r=0.73, p<$

$110.001)$

\section{Discussion}

14 The principal finding of this study was that $W^{\prime}$ influences $P_{\max }$ in physically 15 active individuals that had similar aerobic parameters (LT, CP and $\mathrm{VO}_{2 m a x}$ ). Similar 16 to previous studies (Chidnok et al. 2013; Morton 2011), we have demonstrated 17 that $\mathrm{CP}$ and $\mathrm{W}^{\prime}$ accounted for $\sim 90 \%$ of the variation in $\mathrm{P}_{\max }$, confirming that $\mathrm{CP}$ 18 model can be used to predict different parameters (e.g., Tlim, CP and $\mathrm{P}_{\max }$ ) during 19 INC. Moreover, $\mathrm{CP} / \mathrm{P}_{\max }$ was lower in the $\mathrm{HIGH}$ group and the difference between $20 \mathrm{P}_{\max }$ and CP was significantly related to $\mathrm{W}^{\prime}$. These data confirm and extend the 21 proposal that the $\mathrm{W}^{\prime}$ determines Tlim above $\mathrm{CP}$, with voluntary exhaustion 22 coinciding with both depletion of muscle energy substrates (i.e., [PCr]) and the 23 attainment of $\mathrm{VO}_{2 \max }$ (Chidnok et al. 2013; Vanhatalo et al. 2010).

24 The CP model has been applied to both constant-work rate exercise (CWR) 25 and INC. Morton et al. (1997) have shown that CP and W' estimated during INC 
1 were not different from those derived from conventional method (i.e., CWR). More

2 recently, Chidnok et al. (2013) also found that CP model could accurately predict

3 the performance (i.e., Tlim) during INC. In line with these studies, it was found that

4 the actual $P_{\max }$ for INC was not significantly different from predicted $P_{\max }$ and they

5 were highly correlated. Therefore, these results suggest some important

6 applications. Firstly, it is possible to apply the CP model to estimate different

7 parameters (e.g., Tlim, CP and $P_{\max }$ ) during INC. Moreover, exercise in/tolerance

8 during INC and CWR performed within severe-intensity domain seems to be

9 determined by shared putative physiological mechanisms. Thus, these exercise

10 protocols (INC and CWR) could provide similar insights into the nature of exercise

11 intolerance in health and disease. Finally, CP was the best predictor of $P_{\max }$.

12 Interestingly, Greco et al. (2012) verified in untrained subjects that CP expressed

13 as a unit of metabolic rate (i.e., $\mathrm{VO}_{2}$ ) was highly correlated $(r=0.98)$ with $\mathrm{VO}_{2 \max }$,

14 suggesting that these variables could be determined by similar mechanisms. Thus,

15 the different units utilized to express $\mathrm{CP}, \mathrm{P}_{\max }$ and $\mathrm{VO}_{2 \max }$, could explain the lower

16 relationship between $\mathrm{P}_{\max }$ and $\mathrm{VO}_{2 \max }$ observed in the present study. As

17 mechanical variables, $\mathrm{CP}$ and $\mathrm{P}_{\max }$ are determined by metabolic rate and exercise

18 economy, while $\mathrm{VO}_{2 \max }$ expresses only the former.

19 Several variables (e.g., $\mathrm{VO}_{2 \max }$, exercise economy and $\mathrm{VO}_{2}$ kinetics) have

20 been utilized to explain the inter-individual variability of $P_{\max }$. However, the actual

21 contribution for some of them is still obscure (e.g., anaerobic capacity and muscle

22 power) (Jones and Carter 2000). There is a consensus that $P_{\max }$ reflects the

23 interaction between $\mathrm{VO}_{2 \max }$ and exercise economy. However, some recent

24 interventional studies have challenged the contribution of $\mathrm{VO}_{2 \max }$ and exercise

25 economy to explain the individual variability of $P_{\max }$. In trained cyclists, Rønnestad 
1 et al. (2014) have shown that $P_{\max }$ was increased after a strength-training period,

2 while both $\mathrm{VO}_{2 \max }$ and gross efficiency were unchanged. In addition, Sawyer et al.

3 (2014) found that $\mathrm{P}_{\max }(\sim 8 \%)$, Tlim at severe-intensity domain (39\%) and W' (

$4 \quad 40-60 \%$ ) were improved after 8 weeks of strength training, whereas CP and

$5 \mathrm{VO}_{2 \max }$ were not significantly modified. Our data are in line with these studies, 6 demonstrating that Tlim during severe-intensity exercise (CWR and INC) can be

7 modified with no corresponding changes in aerobic parameters $\left(\mathrm{VO}_{2 \max }\right.$ and $\left.\mathrm{CP}\right)$.

8 In these conditions, $W^{\prime}$ is a determinant of Tlim, and consequently, $P_{\max }$ during

9 INC. Despite the interesting findings presented herein, the physiological basis of

10 W' remains controversial (Dekerle et al. 2006; Vanhatalo et al. 2010). Our results

11 add support to the suggestion that W' may be related to the magnitude (i.e., the 12 relative distance between $\mathrm{CP}$ and $\mathrm{VO}_{2 \max }$ ) of the severe-intensity domain.

13 However, the magnitude of W' has also been associated with the accumulation of 14 metabolites (i.e., $\left[\mathrm{P}_{\mathrm{i}}\right]$ and $\left[\mathrm{H}^{+}\right]$) related with muscle fatigue (Vanhatalo et al. 2010)

15 and / or the amplitude of the $\mathrm{VO}_{2}$ slow component (Murgatroyd et al. 2011). Using 16 an elegant design (i.e., blood flow occlusion), Broxterman et al. (2015) 17 demonstrated that rather than representing an anaerobic capacity (i.e., a constant 18 store of anaerobic energy), the W' can vary across $\mathrm{O}_{2}$ delivery conditions and 19 might be determined by several mechanisms. Thus, future studies are necessary 20 to elucidate the nature of W'.

21 It can be concluded that $W^{\prime}$ influences the $P_{\max }$ reached during INC in 22 physically active individuals with similar aerobic parameters ( $L T, C P$ and $\mathrm{VO}_{2 \max }$ ).

23 Moreover, the $\mathrm{P}_{\max }$ for INC can be predicted by $\mathrm{CP}$ model (i.e., CP and W'). Thus, 24 the depletion of $\mathrm{W}^{\prime}$ and consequently the attainment of $\mathrm{VO}_{2 \max }$ seems to influence 25 the exercise tolerance during INC. 


\section{References}

Bentley, D.J., and McNaughton, L.R. 2003. Comparison of $\mathrm{W}_{\text {peak, }} \mathrm{VO}_{2 \text { peak }}$ and the ventilation threshold from two different incremental exercise tests: relationship to endurance performance. J. Sci. Med. Sport 6(4): 422-435.

Billat, V., Lepretre, P.M., Heugas, A.M., Laurence, M.H., Salim, D., and Koralsztein, J.P. 2003. Training and bioenergetic characteristics in elite male and female Kenyan runners. Med. Sci. Sports Exerc. 35(2): 297-304; discussion 305-306. doi:10.1249/01.MSS.0000053556.59992.A9

Broxterman, R.M., Ade, C.J., Craig, J.C., Wilcox, S.L., Schlup, S.J., and Barstow, T.J. 2015. Influence of blood flow occlusion on muscle oxygenation characteristics and the parameters of the power-duration relationship. J. Appl. Physiol. 118(7): 880-889. doi: 10.1152/japplphysiol.00875.2014

Caputo, F., and Denadai, B.S. 2008. The highest intensity and the shortest duration permitting attainment of maximal oxygen uptake during cycling: effects of different methods and aerobic fitness level. Eur. J. Appl. Physiol. 103(1): 47-57. doi:10.1007/s00421-008-0670-5

Carter, H., Jones, A.M., Barstow, T.J., Burnley, M., Williams, C.A., and Doust, J.H. 2000. Oxygen uptake kinetics in treadmill running and cycle ergometry: a comparison. J. Appl. Physiol. 89(3): 899-907.

Chidnok, W., Dimenna, F.J., Bailey, S.J., Wilkerson, D.P., Vanhatalo, A., and Jones, A.M. 2013. Effects of pacing strategy on work done above critical power during highintensity exercise. Med. Sci. Sports Exerc. 45(7): 1377-1385. doi:10.1249/MSS.0b013e3182860325

Dekerle, J., Brickley, G., Hammond, A.J.P., Pringle, J.S.M., and Carter, H. 2006. Validity of the two-parameter model in estimating the anaerobic work capacity. Eur. J. Appl. Physiol. 96(3): 257-264. doi: 10.1007/s00421-005-0074-8

Greco, C.C., Caritá, R.A., Dekerle, J., and Denadai, B.S. 2012. Effect of aerobic training status on both maximal lactate steady state and critical power. Appl. Physiol. Nutr. Metab. 37(4): 736-743. doi:10.1139/h2012-047

Green, H.J., Burnett, M., Carter, S., Jacobs, I., Ranney, D., Smith, I., and Tupling, S. 2013. Role of exercise duration on metabolic adaptations in working muscle to short-term moderate-to-heavy aerobic-based cycle training. Eur. J. Appl. Physiol. 113(8): 19651978. doi:10.1007/s00421-013-2621-z

Jones, A.M., and Carter, H. 2000. The effect of endurance training on parameters of aerobic fitness. Sports Med. 29(6): 373-386.

Jones, A.M., Vanhatalo, A., Burnley, M., Morton, R.H., and Poole, D.C. 2010. Critical power: implications for determination of $\mathrm{VO}_{2} \max$ and exercise tolerance. Med. Sci. Sports Exerc. 42(10): 1876-1890. doi:10.1249/MSS.0b013e3181d9cf7f

Marsh, A.P., and Martin, P.E. 1997. Effect of cycling experience, aerobic power, and power output on preferred and most economical cycling cadences. Med. Sci. Sports Exerc. 29(9): 1225-1232.

McNaughton, L.R., Roberts, S., and Bentley, D.J. 2006. The relationship among peak power output, lactate threshold, and short-distance cycling performance: effects of incremental exercise test design. J. Strength Cond. Res. 20(1): 157-161. doi:10.1519/R-15914.1

Morton, R.H. 2011. Why peak power is higher at the end of steeper ramps: an explanation based on the "critical power" concept. J. Sports Sci. 29(3): 307-309. doi:10.1080/02640414.2010.534809 
1 Morton, R.H., Green, S., Bishop, D., and Jenkins, D.G. 1997. Ramp and constant power

2 trials produce equivalent critical power estimates. Med. Sci. Sports Exerc. 29(6): 833-

3836.

4 Murgatroyd, S.R., Ferguson, C., Ward, S.A., Whipp, B.J., and Rossiter, H.B. 2011.

5 Pulmonary $\mathrm{O}_{2}$ uptake kinetics as a determinant of high-intensity exercise tolerance in

6 humans. J. Appl. Physiol. 110(6): 1598-1606. doi: 10.1152/japplphysiol.01092.2010

7 Rønnestad, B.R., Hansen, J., Hollan, I., and Ellefsen, S. 2015. Strength training improves

8 performance and pedaling characteristics in elite cyclists. Scand. J. Med. Sci. Sports.

9 25(1): e89-98. doi:10.1111/sms.12257

10 Sawyer, B.J., Stokes, D.G., Womack, C.J., Morton, R.H., Weltman, A., and Gaesser, G.A.

11 2014. Strength training increases endurance time to exhaustion during high-intensity

12 exercise despite no change in critical power. J. Strength Cond. Res. 28(3): 601-609.

13 doi:10.1519/JSC.0b013e31829e113b

14 Vanhatalo, A., Fulford, J., DiMenna, F.J., and Jones, A.M. 2010. Influence of hyperoxia

15 on muscle metabolic responses and the power-duration relationship during severe-

16 intensity exercise in humans: a ${ }^{31} \mathrm{P}$ magnetic resonance spectroscopy study. Exp.

17 Physiol. 95(4): 528-540. doi:10.1113/expphysiol.2009.050500

18 Wilkerson, D.P., Burnley, M., Koppo, K., and Jones, A.M. 2003. Prior heavy exercise

19 increases the time to exhaustion during subsequent peri-maximal exercise in humans.

20 J. Physiol. [abstract] 547P: PC15.

21 Zuniga, J.M., Housh, T.J., Camic, C.L., Bergstrom, H.C., Traylor, D.A., Schmidt, R.J., and 22 Johnson, G.O. 2012. Metabolic parameters for ramp versus step incremental cycle 23 ergometer tests. Appl. Physiol. Nutr. Metab. 37(6): 1110-1117. doi:10.1139/h2012$24 \quad 098$

25 
1 Table 1 - Physiological and mechanical parameters obtained during tests.

\begin{tabular}{lccc}
\hline Variables & Overall & HIGH & LOW \\
& $(\mathbf{N}=\mathbf{3 0})$ & $\mathbf{( N = 1 0 )}$ & $\mathbf{( N = 1 0 )}$ \\
\hline $\mathrm{VO}_{2 \max }\left(\mathrm{L} \cdot \mathrm{min}^{-1}\right)$ & $3.58 \pm 0.40$ & $3.84 \pm 0.50$ & $3.49 \pm 0.37$ \\
$\mathrm{P}_{\max }(\mathrm{W})$ & $317 \pm 33$ & $337 \pm 23$ & $299 \pm 40^{*}$ \\
$\mathrm{LT}(\mathrm{W})$ & $103 \pm 26$ & $92 \pm 21$ & $102 \pm 17$ \\
$\mathrm{CP}(\mathrm{W})$ & $206 \pm 22$ & $213 \pm 22$ & $200 \pm 29$ \\
$\mathrm{CP} / \mathrm{P}_{\max }(\%)$ & $65.0 \pm 3.1$ & $63.2 \pm 3.8$ & $66.8 \pm 2.5^{*}$ \\
$\mathrm{~W}^{\prime}(\mathrm{kJ})$ & $20.3 \pm 4.3$ & $25.1 \pm 2.9$ & $15.9 \pm 1.8^{*}$
\end{tabular}

2 HIGH: high W' group; LOW: low W' group; $\mathrm{VO}_{2 \max }$ : maximal oxygen uptake; $\mathrm{P}_{\max }$ :

3 maximal power output; LT: lactate threshold; CP: critical power; CP/P max: critical

4 power relative to maximal power output; W': curvature constant of the power-time

5 relationship. * Significantly different between HIGH and LOW $(p<0.05)$.

6 


\section{Figure Caption}

2

3 FIGURE 1. Actual versus predicted maximal power output $\left(P_{\max }\right)$ during ramp

4 incremental test. Prediction was made using parameters derived from the power-

5 time relationship (i.e., CP and W') (Morton 2011). The solid line is the best-fit linear

6 regression, and the dashed line is the line of identity. 


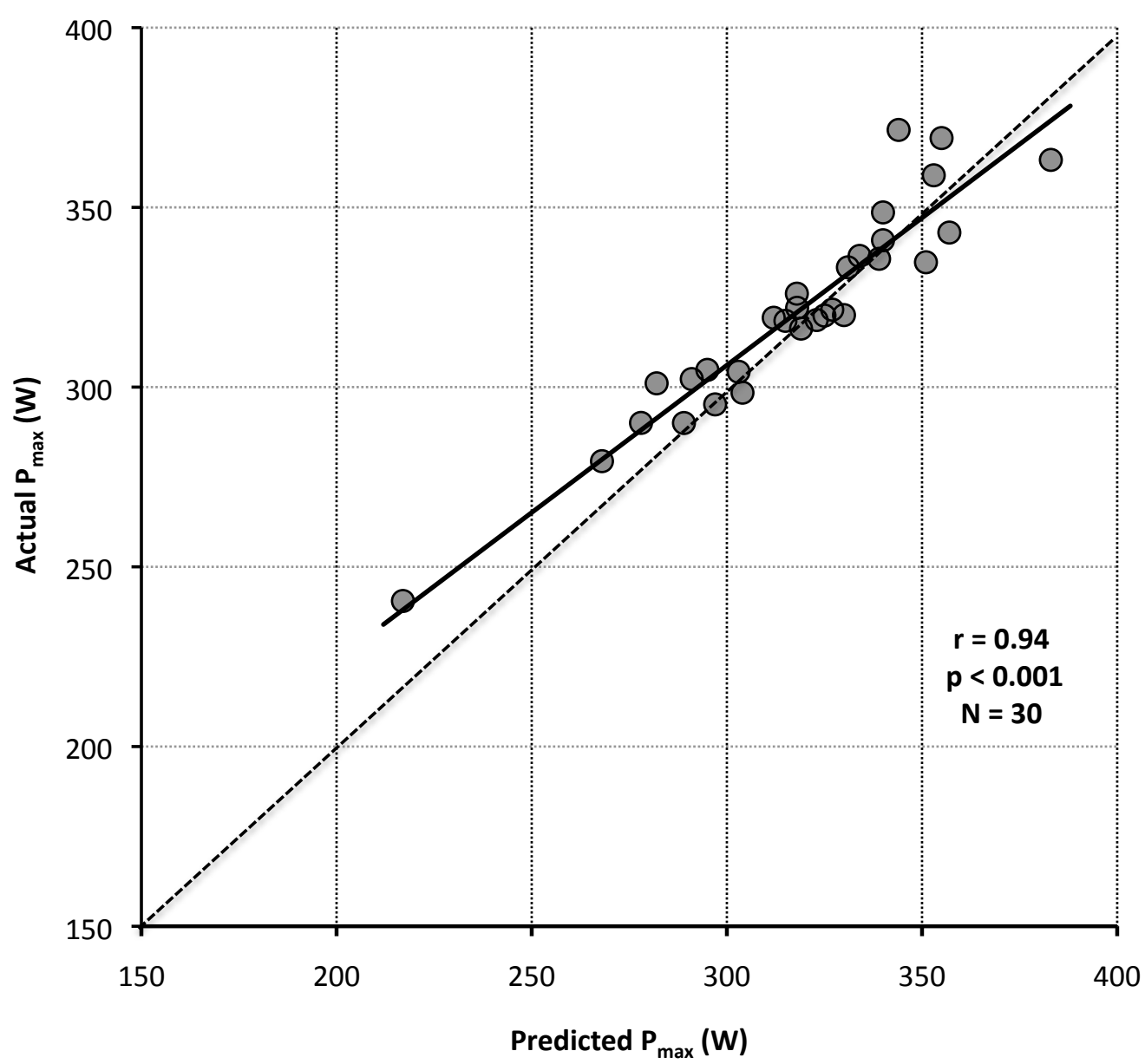

https://mc06.manuscriptcentral.com/apnm-pubs 